OPEN ACCESS

Edited by:

Binghui Li,

Capital Medical University, China

Reviewed by:

Jun Fan,

Jinan University, China

Guoliang Qing,

Wuhan University, China

*Correspondence:

Peng Jiang

pengjiang@tsinghua.edu.cn

${ }^{t}$ These authors have contributed

equally to this work

Specialty section:

This article was submitted to

Molecular Medicine

a section of the journal

Frontiers in Cell and Developmental

Biology

Received: 21 April 2021

Accepted: 14 May 2021

Published: 10 June 2021

Citation:

Zhao L, Xia W and Jiang P (2021) CREB1 and ATF1 Negatively Regulate Glutathione Biosynthesis Sensitizing

Cells to Oxidative Stress.

Front. Cell Dev. Biol. 9:698264.

doi: 10.3389/fcell.2021.698264

\section{CREB1 and ATF1 Negatively Regulate Glutathione Biosynthesis Sensitizing Cells to Oxidative Stress}

\author{
Lina Zhao ${ }^{\dagger}$, Wenjun Xiat and Peng Jiang* \\ School of Life Sciences, Tsinghua University, Beijing, China
}

The cAMP response element binding protein (CREB) family activating transcription factor 1 (ATF1) and cAMP response element binding protein 1 (CREB1) have been reported in a diverse group of tumors, however, the mechanistic basis for this remains unclear. Here we found that CREB1 and ATF1 unexpectedly regulate glutathione (GSH) biosynthesis by suppressing the expression of glutamate-cysteine ligase modifier subunit (GCLM) and glutathione synthase (GSS), two key enzymes of GSH biosynthesis pathway. Mechanistic studies reveal that GCLM and GSS are direct transcriptional targets of CREB1 and ATF1. Through repressing the expression of these two enzymes, CREB1 and ATF1 reduce the GSH biosynthesis and the capability of cells to detoxicate reactive oxygen species (ROS), thereby increasing cellular susceptibility to oxidative stress. Therefore, our findings link CREB1 family to cellular metabolism, and uncover a potential therapeutic approach by targeting GCLM or oxidative stress for the treatment of tumors with relatively high expression of CREB1 family proteins.

Keywords: CREB1, ATF1, GSH, ROS, survival, proliferation

\section{INTRODUCTION}

Reactive oxygen species (ROS) is an important determinant of cancer cells metabolism phenotypes (Rodic and Vincent, 2018). The outcomes of ROS performance in tumor cells are dose-dependent. In general, low level of ROS facilities proliferation, growth, invasion and metastasis of tumor cells, while relatively high level of ROS leads to cell cycle arrest and even cell death. Therefore, it is essential for cancer cells to optimize cellular ROS level to maintain tumor progression (Ahmad et al., 2005; Aykin-Burns et al., 2009; Rodic and Vincent, 2018). To achieve this, tumors cells could increase their antioxidative capacity by upregulating relevant metabolic molecules and the expression of certain antioxidant enzymes. Glutathione (GSH) is thought to be a main small thiol-antioxidant derivative (Veeravalli et al., 2011; Quintana-Cabrera et al., 2012), consisting of L-glutamine, L-cysteine, and L-glycine, found in all mammalian tissues (Lu, 2009). GSH is generally synthesized exclusively in the cytoplasm in two consistently steps at the expenditure of ATP, the first step, which is also a rate-limiting step, catalyzes the generation of $\gamma$-glutamylcysteine by the GCL, and the glutathione synthase (GSS) catalyzes the second step to produce GSH. The GCL holoenzyme consists of two separate proteins, a catalytic subunit (GCLC) and a modifier subunit (GCLM) (Krejsa et al., 2010), which are encoded by different genes in humans. GCLC exhibits 
catalytic activity (Seelig et al., 1984; Lu, 2009) and GCLM is enzymatically inactive but plays an important regulatory role by lowering the $\mathrm{Km}$ of GCL for glutamate and raising the Ki for GSH (Huang et al., 1993a,b). GSH synthesis pathway is severely activated by some stimuli including oxidative stress.

cAMP response element binding protein 1 (CREB1) belongs to the bZIP transcription factors. The other two members of CREB1 family are activating transcription factor 1 (ATF1) and cAMP response element modulator (CREM). CREB1 and ATF1 both express ubiquitously, whereas CREM mainly expresses in neuroendocrine tissues (Mayr and Montminy, 2001). Although CREB1 has been implicated in the physiology of nerve and most recently in some cancer cells by cooperating with some protein kinases or small molecules, including PKA, PKB (AKT), MAPK, cAMP, and $\mathrm{Ca}^{2+}$ to inhibit apoptosis and promote cell survival in unstressed situation (Bonni and Brunet, 1999; Shankar et al., 2005; Chhabra et al., 2007; Pigazzi et al., 2007; Aggarwal et al., 2008; Shukla et al., 2009), whether CREB families play a role in modulating tumor cellular metabolism remains unknown.

\section{RESULTS}

To determine if CREB1 regulates GSH synthesis, we examined the effect of CREB1 silencing on the expression of several major enzymes of the GSH synthetic pathways in multiple cell lines including U2OS and U87 cells (Figure 1A). Interestingly, while the mRNA level of most enzymes remained approximately the same (CTH and GCLC) or changed inconsistently (CBS, CDO1, and CSD), the mRNA levels of GCLM and GSS increased consistently and significantly when CREB1 was knocked down in U2OS, U87, and HepG2 cells (Figures 1B,C and Supplementary Figure 1A). Similar findings were found in A549, H1299, and H1975 cells. CREB1 ablation led to an increase in the expression of GCLM and GSS expression (Supplementary Figures 1BD). Like CREB1, knocking down of ATF1 expression in U2OS and U87 cells resulted in strong increase in mRNA levels of both GCLM and GSS (Figures 1D,E). Moreover, upon ATF1 knockdown, GCLM and GSS mRNA levels augmented in multiple cell lines including HepG2, A549, H1299, and H1975 cells (Supplementary Figures 1E-H). Results from western blot analysis revealed that silencing of either of CREB1, ATF1 or both enhanced the protein levels of GCLM and GSS in U2OS and U87 cells, as well as in HepG2 and A549 cells (Figure 1F and Supplementary Figures 1I,J). Similarly, sgRNA-mediated knockout of CREB1 and ATF1 in U2OS cells strongly boosted the expression of GCLM and GSS (Supplementary Figure 1K). These findings were further confirmed by overexpression of CREB1 and ATF1. In line with the knockdown data, enforced expression of CREB1 and ATF1 led to strong reduction in protein levels of GCLM and GSS in U2OS cells (Supplementary Figure 1L). Collectively, these findings show that both CREB1 and ATF1 negatively regulate the expression of GCLM and GSS, two key enzymatic steps of GSH synthesis pathway.

To investigate whether CREB1 and ATF1 are the transcription factors for GCLM and GSS, we analyzed human GCLM and GSS gene sequences for potential response elements of CREB family proteins, which share the conservative sequence of $5^{\prime}$ TGACGTCA-3'. Potentially, we identified a response element (RE) for CREB1 and ATF1, respectively, in each of them (Figure 2A). Chromatin immunoprecipitation (ChIP) assays showed that CREB1 and ATF1 could bind to these REs (Figures 2B,C). Next, we cloned the fragments of GCLM and GSS genes containing the corresponding response elements into the promotor region of a luciferase reporter plasmid, and found that CREB1 and ATF1 repressed the luciferase activity driven by these REs, but not the mutant ones (Figures 2D,E). These results together indicate that GCLM and GSS may be the transcriptional targets for CREB1 and ATF1.

GCLM and GSS are essential for GSH synthesis (Li et al., 2016). Using small interfering RNA (siRNA) to simultaneously knock down the expression of CREB1 and ATF1, we observed that GSH production was substantially increased in U2OS cells (Figures $\mathbf{3 A}, \mathbf{B}$ ). GSH is considered to be one of the most important scavengers of reactive oxygen species (ROS). Consistent with this, ablation of CREB1 and ATF1 decreased the cellular level of ROS in both U2OS, U87, and HepG2 cells as determined by DCF staining (Figure 3C and Supplementary Figures 2A,B). The specificity of the assay was shown by an increase in the detected ROS upon G6PD silencing (Supplementary Figure 2C). G6PD is the first and ratelimiting enzyme of the PPP pathway, which is the main source of intracellular antioxygen NADPH (nicotinamide adenine dinucleotide phosphate, reduced) (Jiang et al., 2011). Conversely, overexpression of CREB1 and ATF1 led to increased intracellular ROS in U2OS cells (Supplementary Figure 2D). Consistent with previous study (Huang et al., 1993b; Chhabra et al., 2007), GSS depletion showed no effect on ROS level, which could be due to the complex GSH-independent action(s) of GSS (Supplementary Figure 2E). Nevertheless, silencing GCLM resulted in an increase in ROS level (Supplementary Figure 2E). Moreover, knockdown of GCLM alone elevated intracellular level of ROS, and reversed the suppressive effect of depletion of CREB1 and ATF1 on ROS in U2OS cells (Figure 3D). Collectively, these results indicate that CREB1 and ATF1 have a role in maintaining ROS though downregulation of GCLM and GSS expression.

The above findings that CREB1 and ATF1 unexpectedly reduce cell capability to scavenge ROS promoted us to investigate whether cells with ablation of CREB1 and ATF1 are resistant to oxidative stress. Indeed, deletion of CREB1 and ATF1 in U2OS cells resulted in a reduction in cell death as determined by FACS analysis when cells were cultured in medium containing $100 \mu \mathrm{M} \mathrm{H}_{2} \mathrm{O}_{2}$ (Figure 4A). To examine whether GCLM and GSS are involved in CREB1- and ATF1-mediated cell death under oxidative stress, we knocked down the expression of GCLM and GSS in U2OS cells expressing siRNAs against CREB1 and ATF1. Again, depletion of CREB1 and ATF1 led to increased cells viability, and this effect was reversed by GCLM and GSS silencing in the presence of $100 \mu \mathrm{M} \mathrm{H} \mathrm{H}_{2} \mathrm{O}_{2}$ (Figures $4 \mathrm{~B}, \mathrm{C}$ ). We next confirmed these findings by an anchorage-independent growth in soft agar, an in vitro measurement of tumorigenicity. Depletion of CREB1 and ATF1 caused increased capacity of U87 cells to form colonies in soft agar supplied with $100 \mu \mathrm{M} \mathrm{H}_{2} \mathrm{O}_{2}$, 


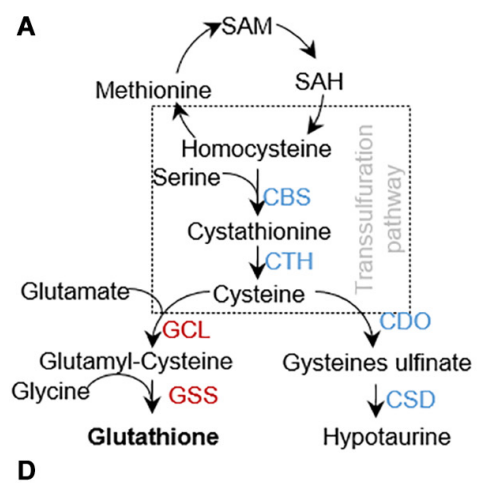

D

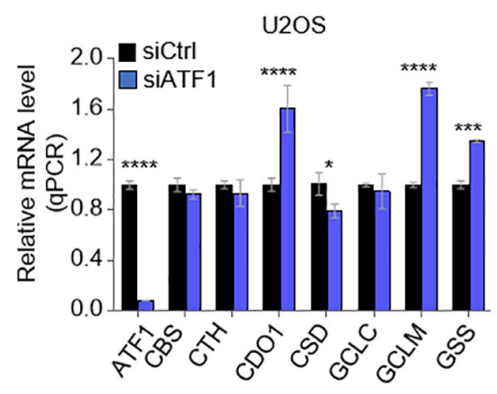

B

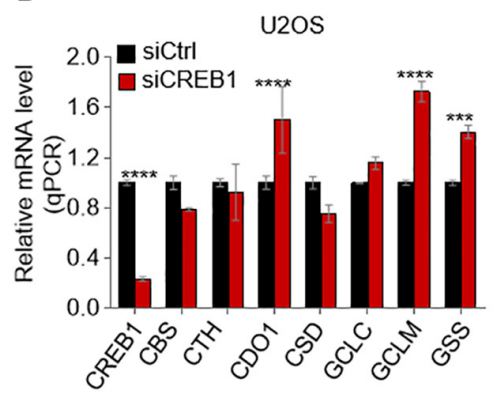

E

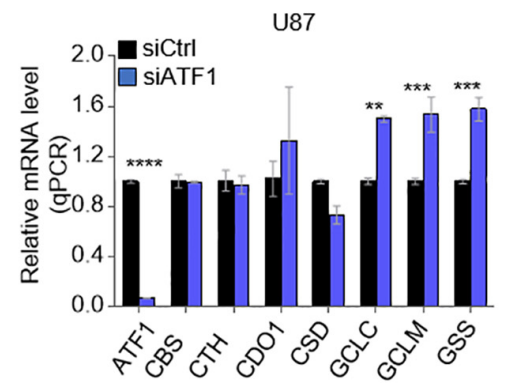

C

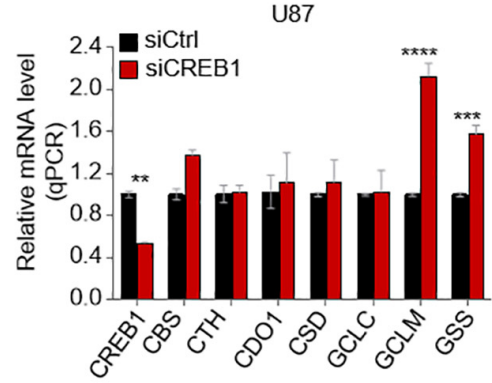

$\mathbf{F}$

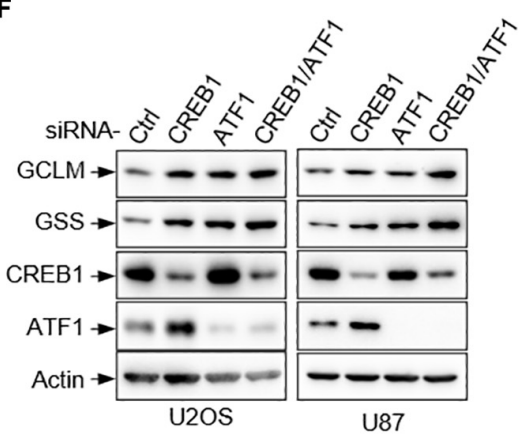

FIGURE 1 | CREB1 and ATF1 repress the expression of GCLM and GSS. (A) Schematic depicting the GSH synthesis pathway and its associated hypotaurine synthesis pathway. (B,C) Relative mRNA levels of enzymes of GSH synthesis pathway in U2OS cells (B) and U87 cells (C) transfected with CREB1 siRNA or control siRNA. (D,E) Relative mRNA levels of GSH synthesis enzymes in U2OS cells (D) and U87 cells (E) transfected with control siRNA or ATF1 siRNA as indicated. (F) U2OS and U87 cells expressing control siRNA, or siRNAs against CREB1, ATF1, or both (CREB1/ATF1) as indicated. Protein expression was analyzed by Western blot. Data are means $\pm \mathrm{SD} .(n=3),{ }^{\star} p<0.05,{ }^{* \star} p<0.01,{ }^{\star \star \star} p<0.001,{ }^{\star \star \star \star *} p<0.00001$.

and knockdown of GCLM and GSS almost totally reduced the anchorage-independent growth regardless of CREB1 and ATF1 status (Figures 4D,E and Supplementary Figure 3).

\section{CONCLUSION}

In this work, we found a previously unappreciated role for CREB family proteins in controlling GSH synthesis. Through downregulation of the expression of GCLM and GSS, CREB1 and ATF1 unexpectedly reduce GSH generation and thereby dampen the cellular ability to scavenge ROS, leading to increased susceptibility of cells to oxidative stress. Intriguingly, GSS seems not to be essential for ROS detoxification, but still acts a key metabolic enzyme of the GSH de novo synthetic pathway. To elucidate the underlying mechanism(s) for these unexpected observations would be of great interest. One potential expectation is that GSS possess ROS-related but GSH synthesis-independent functions, which could comprise the effect of GSH production on ROS. Nevertheless, GCLM downregulation appears to be sufficient to abolish ROS reduction in cells depleted of CREB family proteins CREB1 and ATF1. CREB1 has been implicated in supporting tumor cell proliferation and survival in some types of tumor cells, indicating that CREB1 might be an oncogenic protein that can help tumor cell to proliferate in certain situations (Thway and Fisher, 2012). Here our findings reveal a metabolic mechanism for CREB1-mediated tumor cell survival, and also suggest that targeting GCLM or oxidative stress potentially could be a therapeutic approach for treatment of tumors with relatively high expression of CREB1 family proteins.

\section{MATERIALS AND METHODS}

\section{Antibodies and Reagents}

Antibodies against the following proteins/epitopes were used for immunoblot with the sources, catalog numbers, and dilutions indicated: Actin (Easy Bio, BE0037-10, 1:5000), Flag (Sigma-Aldrich, F3165, 1:5000), GCLM (Proteintech, 142411-AP, 1:1000), GSS (Santa Cruz Biotechnology, sc-166882, 1:1000), CREB1 (Proteintech, 12208-1-AP, 1:1000), and ATF1 (Proteintech, 11946-1-AP, 1:1000).

The ANNEXIN V-FITC/PI Apoptosis Detection Kit were purchased from Solarbio. The following regents were purchased from Sigma-Aldrich: crystal violet (CV), 0.4\% trypan blue solution, $2^{\prime}, 7^{\prime}$-dichlorofluorescin diacetate (DCF), propidium iodide (PI), puromycin.

\section{Cell Culture}

Cells were maintained in standard culture conditions without any antibiotic. A549, H1299, HEPG2, H1975, and U87 were from ATCC (Manassas, VA, United States). All cells were cultured in a $5 \% \mathrm{CO}_{2}$ humidified incubator (Thermo Fisher Scientific, United States) at $37^{\circ} \mathrm{C}$. A549 cell line was maintained in standard 
A

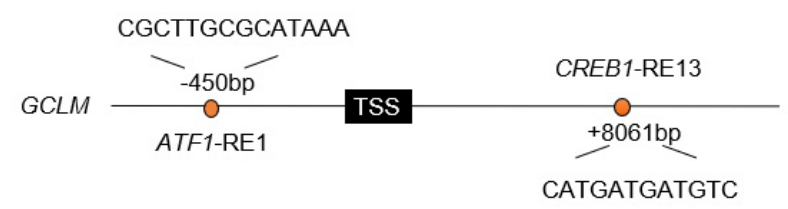

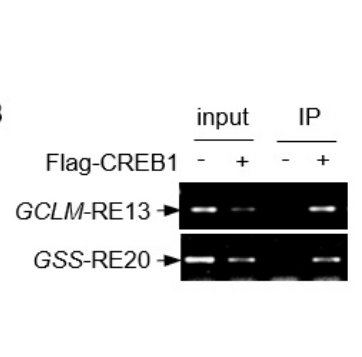
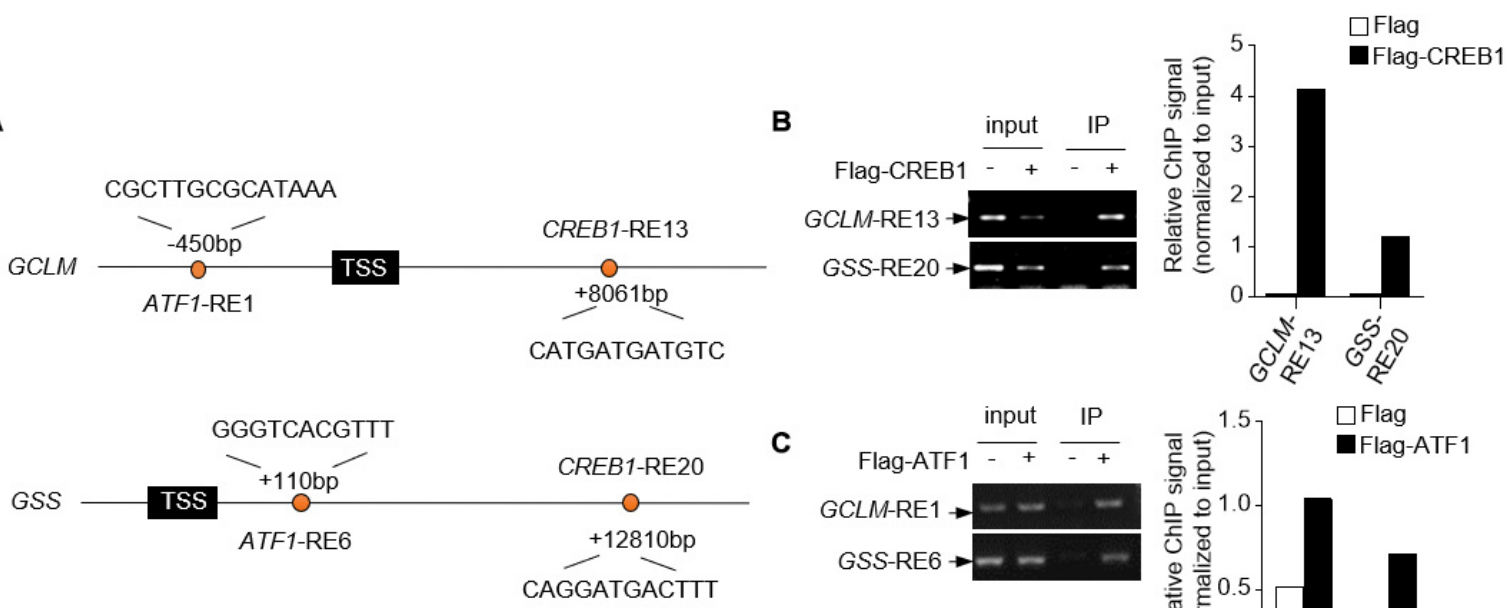

Flag-ATF1 $\frac{\text { input }}{-+} \frac{\text { IP }}{-+}$
GCLM-RE1 $\rightarrow-\infty$
GSS-RE6 $\rightarrow-\infty$

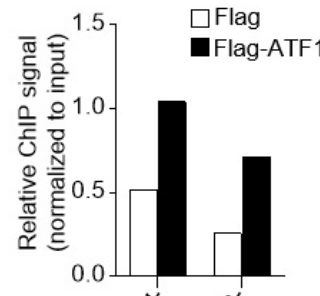

$\square$ Flag

- Flag-ATF1

E

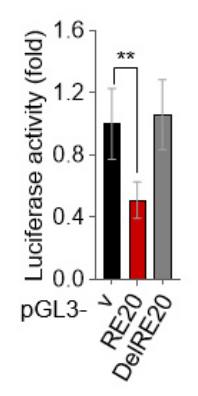

$\mathrm{E}$
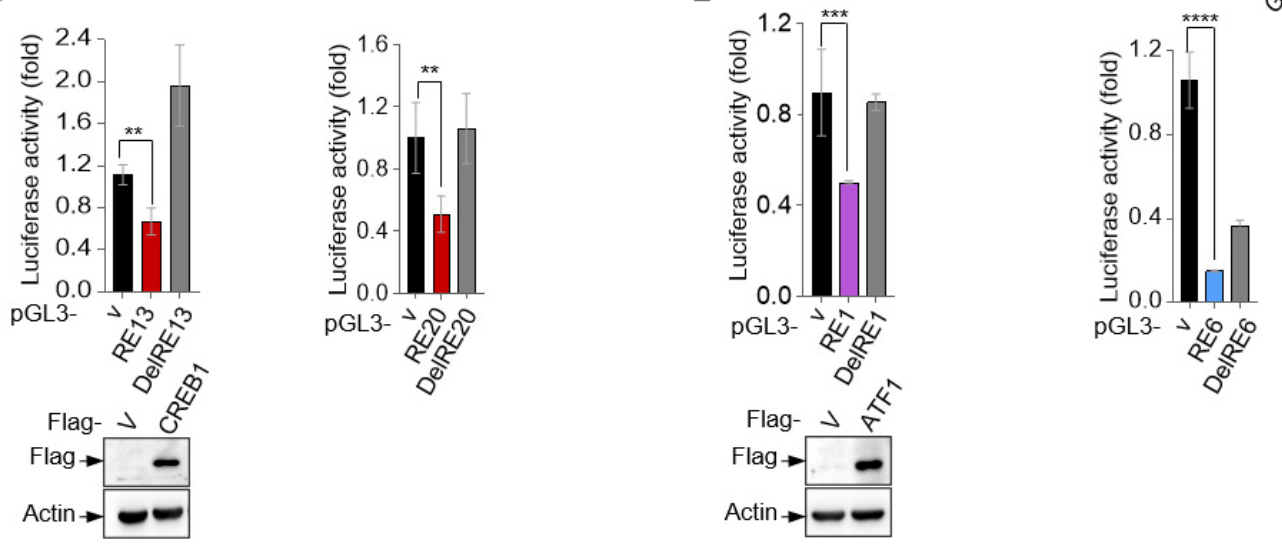

FIGURE 2 | GCLM and GSS are transcriptional targets of CREB1 and ATF1. (A) Schematic representation of human GCLM and GSS genomic structure. The sequences of potential CREB1 and ATF1 response elements GCLM-RE13, GSS-RE20, GCLM-RE1, and GSS-RE6 are shown. (B,C) 293T cells were transfected with Flag-CREB1 (B), Flag-ATF1 (C) or vector control plasmid. Cell lysates were used for ChIP assay using anti-Flag antibody. Bound DNA was amplified by PCR. Results are representative of three independent experiments. (D,E) Luciferase constructs containing indicated responsive elements (REs) were transfected into $293 T$ cells together with Flag-CREB1 (D), Flag-ATF1 (E) and vector control, respectively. Renilla vector pRL-CMV was used as a transfection internal control. The relative luciferase activity was normalized to the co-transfected Renilla activity. Data are means $\pm \mathrm{SD}$. $(n=3),{ }^{\star \star} p<0.01,{ }^{\star \star \star} p<0.001,{ }^{\star \star \star \star} p<0.0001$.

Dulbecco's modified Eagle's medium (DMEM) (Thermo Fisher Scientific, C11995500BT) with $10 \%$ fetal bovine serum (FBS) (GEMINI, 100-106). U2OS cells were cultured in McCoy's 5 A Medium, U87 and HEPG2 cells in MEM supplemented with nonessential amino acid (Gibco,11140050). H1299 and H1975 cells were cultured in standard RPMI1640 medium (Thermo Fisher Scientific, 11875093) with 10\% FBS. Unless indicated otherwise, all cells were cultured without the addition of penicillinstreptomycin and for no more than two consecutive months, and were routinely examined for mycoplasma contamination.

\section{Genes Knock Down With siRNA}

The flowing siRNAs were purchased from GenePharma. The sequences were: CREB1\#1, 5'-AAA CAUUAACCAUGACCAATT- $3^{\prime}$; CREB1\#2, 5'-GGUGCCAA CUCCAAUUUACTT-3'; ATF1\#1, 5'-AGGUACAACUAUUC
UUCAGUA-3'; ATF1\#2, 5' -GCUCAACAGGUAUCAUCUUUA3'; GCLM; 5'-GCAAGUUUCCAAGAAGCUCTT-3'; GSS, $5^{\prime}$ - CCAUCAAACAGGAUGACUUUATT $-3^{\prime}$ G6PD, 5' ACGAGCUGAUGAAGAGAGUGGGUUU- $3^{\prime}$. siRNAs were transfected into cells using Lipo2000 Transfection Agent (Thermo Fisher Scientific, 12566014) following the manufacturer's instruction.

\section{Plasmids}

The coding sequences corresponding to the full-length human CREB1 and ATF1 were amplified by polymerase chain reaction (PCR) from cDNA library of $293 \mathrm{~T}$ cells and then cloned into Prk5-flag empty vector as indicated. The cloning sequences are as follows: CREB1, 5'-CGCGGATCCGCGATGACCATGGAATCTGGAGCCG-

$3^{\prime}$ (forward) and 5'-CCCAAGCTTGGGTTAATCTGAT 
A

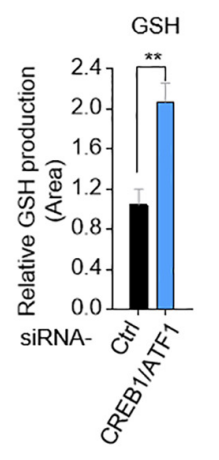

B

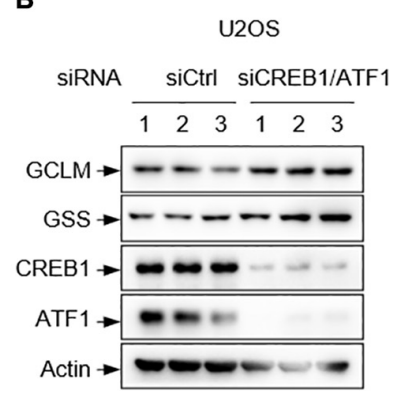

c

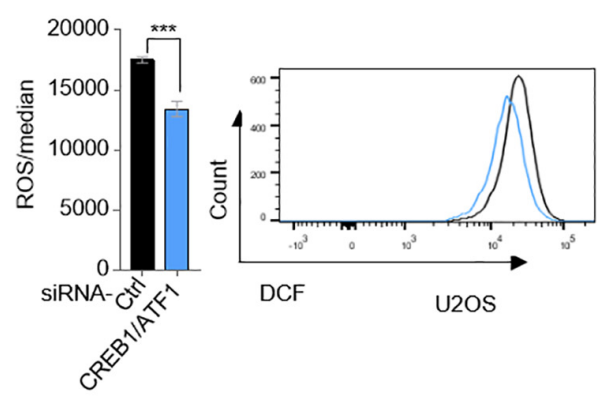

D

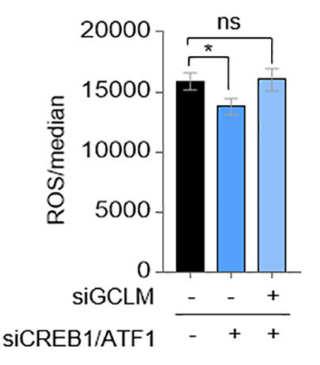

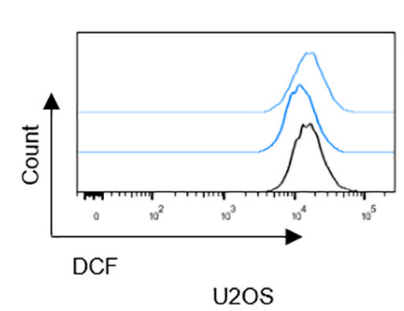

U2OS
U2OS

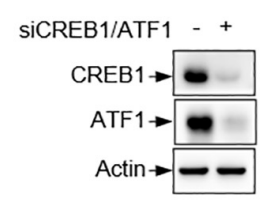

FIGURE 3 | CREB1 and ATF1 inhibit intracellular ROS. (A,B) U2OS cells transfected with control siRNA, or CREB1 siRNA together with ATF1 siRNA (siCREB1/ATF1) were assayed for intracellular GSH production by LC-MS. (C) Relative ROS level in U2OS cells transfected with CREB1 and ATF1 siRNAs (SiCREB1/ATF1), or control siRNA was determined by flow cytometry analysis. Protein expression was analyzed by Western blot. (D) U2OS cells were transfected with control, CREB1, ATF1 and/or GCLM siRNA as indicated. Relative ROS level was analyzed by flow cytometry. Protein expression was analyzed by Western blot. Data are means \pm SD. $(n=3),{ }^{\star} p<0.05,{ }^{\star \star} p<0.01,{ }^{\star \star *} p<0.001$

TTGTGGCAGTAAAGGTCCT-3' (reverse); ATF1, 5'-TGC TCTAGAATGGAAGATTCCCACAAGAGTAC-3' (forward) and $5^{\prime}$-CCCAAGCTTTCAAACACTTTTATTGGAATAAAGAT CC-3' (reverse). sgRNAs were made in px330-GFP vector. The sequences were: CREB1:5'-CACCGGGGCAGACAGTTC

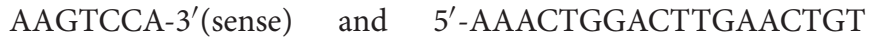
CTGCCCC-3' (antisense); ATF1: 5'-CACCGGAACGCTG ACGTCATCAACCCG-3' (sense) and 5' ${ }^{\prime}$-AAACGCACCGAC TCGGTGCCAC-3' (antisense).

\section{Semi-Quantitative RT-PCR and Quantitative RT-PCR}

RNA was extracted by TRIzol (15596-018, Invitrogen) according to the manufacturer's instruction. $2 \mu \mathrm{g}$ RNA of each sample was reversed to complementary DNA by First-strand cDNA Synthesis System (Thermo Fisher Scientific, catalog No. K1622), and $0.2 \mu \mathrm{g}$ cDNA was used as a template to perform PCR. Quantitative PCR were performed on CFX96 Real-Time PCR System (Bio-Rad, United States) and the amplifications were done using the SYBR Green PCR Master Mix (Gene Star, China). The primer pairs were: CREB1, 5'-ATTCACAGGAGTCAGTGGATAGT-3' (forward) and 5'-CACCGTTACAGTGGTGATGG-3' (reverse); ATF1, 5'-AGGACTCATCCGACAGCATAG-3' (forward) and 5' -TTCTGCCCCGTGTATCTTCAG-3' (reverse); CBS, 5' -GTCA GACCAAGTTGGCAAAGT-3' (forward) and $5^{\prime}$-CACCCCGA
ACACCATCTGC-3' (reverse); CTH, 5'-AAAGACGCCTCCT CACAAGG-3' (forward) and 5'-AAGGCAATTCCTAGTG GGATTTC-3' (reverse); CDO1, 5'-TCCATTGGCTTACATCG AGTAGA-3' (forward) and 5' -CCCGAAGTTGCATTTGGAGT$3^{\prime}$ (reverse); CSD, 5'-AGAAGCGGGAAGGGTTTGAG-3' (forward) and $5^{\prime}$-CCTTTCGTGGTAATCTGGACTC-3' (re verse); GCLC, 5'-GGAGACCAGAGTATGGGAGTT-3' (fo rward) and 5'-CCGGCGTTTTCGCATGTTG-3' (reverse); GCLM， 5'-CATTTACAGCCTTACTGGGAGG-3' (forward) and 5'-ATGCAGTCAAATCTGGTGGCA-3' (reverse); GSS, $5^{\prime}$-GGGAGCCTCTTGCAGGATAAA-3' (forward) and $5^{\prime}$ GAATGGGGCATAGCTCACCAC- $3^{\prime}$ (reverse); $\beta$-actin, 5'GAC CTGACTGACTACCTCATGAAGAT- $3^{\prime}$ (forward) and $5^{\prime}$ GTCACACTTCATGATGGAGTTGAAGG-3' (reverse). The fold changes of gene expression are normalized to $\beta$-actin.

\section{Western Blot Analysis}

Whole-cell lysates were obtained using modified RIPA lysis buffer (10 mM Tris- $\mathrm{HCl}$ at $\mathrm{pH} 7.5,5 \mathrm{mM}$ EDTA, $150 \mathrm{mM} \mathrm{NaCl}, 1 \% \mathrm{NP}-$ $40,1 \%$ sodium deoxycholate, $0.025 \%$ SDS, and added protease cocktail freshly). Cells were washed and incubated in lysis buffer for $30 \mathrm{~min}$ on ice, and boiled in $5 \mathrm{x}$ loading buffer. Protein samples were resolved by SDS-PAGE and transferred onto nitrocellulose membrane. The membrane was then blocked in 5\% skimmed milk in TBST and probed with the indicated antibodies. 
A

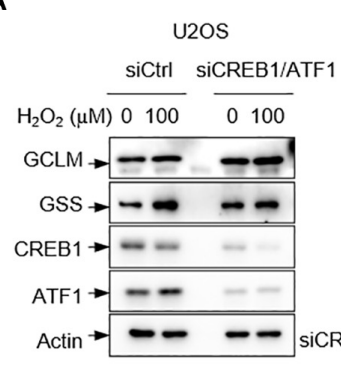

C

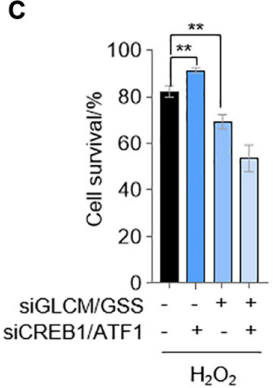

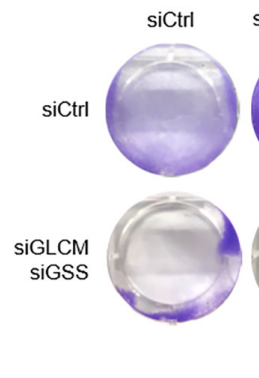

D

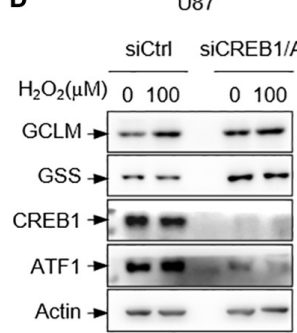

B

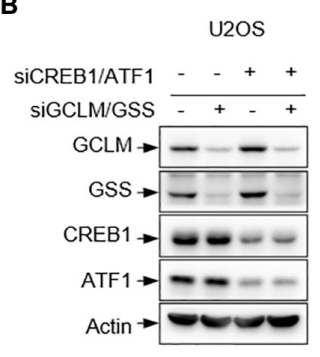

E

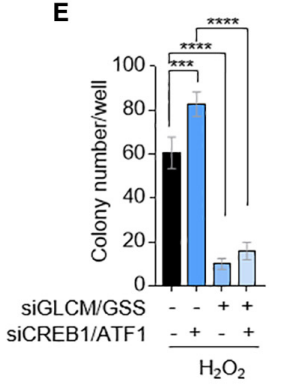

FIGURE 4 | CREB1 and ATF1 inhibit cell survival under oxidative stress. (A) U2OS cells transfected with control, or CREB1 and ATF1 siRNAs (siCREB1/ATF1) were treated with $100 \mu \mathrm{M} \mathrm{H}_{2} \mathrm{O}_{2}$ for $24 \mathrm{~h}$. Protein expression was measured by Western blot analysis. Cell death was analyzed by flow cytometry after PI staining. (B,C) U2OS cells treated with control, CREB1, ATF1, GCLM, and/or GSS siRNAs as indicated. Protein expression was analyzed by Western blot (B), and cell survival was assayed by crystal violet staining (C). (D) U87 cells transfected with control siRNA, or siRNAs against CREB1 and ATF1 as indicated were treated with $100 \mu \mathrm{M}$ $\mathrm{H}_{2} \mathrm{O}_{2}$ for 24 h. Protein expression was analyzed by Western blot. (E) U87 cells were transfected with control, CREB1, ATF1, GCLM, and/or GSS siRNAs for colony formation assay. Numbers of colonies with a diameter greater than $20 \mu \mathrm{m}$ were quantified. Data are means $\pm \mathrm{SD}$. $(n=3)$, ${ }^{\star \star} p<0.01,{ }^{* \star \star} p<0.001$,

${ }^{* \star \star *} p<0.0001$.

\section{Chromatin Immunoprecipitation (ChIP) and Reporter Assays}

We used JASPAR ${ }^{1}$ to identify the potential CREB1 and ATF1 response elements in GCLM and GSS genes. The ChIP analysis was performed with some modifications. The response elements were amplified by PCR. The primer pairs were: GCLM RE1, 5'-CCGCTCGAGGTCCTTGAAGGTAGCAAAGCGA$3^{\prime}$ (forward) and 5'-CCCAAGCTTCGGCCGCCCCAGG GTGGGCTTC-3' (reverse); GCLM RE13, 5'-GAGTCAGAAT AGAACTTCAACTT-3' (forward) and 5'-TCTCTTCATG TAACTTGCCCAG-3' (reverse); GSS RE6, 5'-CCCT CGAGTCTGCCCCGCCTGCTCTTTAA-3' (forward) and 5' CCCAAGCTTCTAGACACTAGTTGCCCCGCTC-3' (reverse); GSS RE20, 5'-GATTCAGGAGTCAATGACTGA-3' (forward) and $5^{\prime}$-GACAGGTTACAGACTGGAGTAG-3' (reverse).

For reporter assay, the sequences for CREB1 response elements in GCLM and GSS gene are: GCLM-RE13, 5'CATGATGATGTC-3', GSS-RE20, 5' -CAGGATGACTTT-3'. The sequences for ATF1 response elements in GCLM and GSS gene are: GCLM-RE1, 5'-CCGCTTGCGCATAAA-3'; GSS-RE6, 5'-GGGTCACGTTT-3'. These binding regions were cloned into pGL3-basic vector (Promega, Madison, WI, United States, catalog No: E1751). The luciferase activity was determined using a Dual Luciferase Assay System (Promega, catalog No: E1910).

${ }^{1}$ http://jaspar.genereg.net
Transfection efficiency was normalized on the basis of the Renilla luciferase activity.

\section{Measurements of GSH and ROS}

Glutathione was determined using liquid chromatography-mass spectrometry (LC-MS). We used cold $80 \%$ methanol to extract metabolites analyzed by LC-MS. The measured mass isotopomer distributions were corrected by natural abundances.

Reactive oxygen species levels were determined as followed. Cells were incubated at $37^{\circ} \mathrm{C}$ for $30 \mathrm{~min}$ in PBS containing $10 \mu \mathrm{M} 2^{\prime}, 7^{\prime}$-dichlorodihydrofluorescein diacetate (H2-DCFDA, Sigma). Afterward, the cells were washed twice in PBS, treated with trypsin, and resuspended in PBS. Fluorescence was immediately measured using a FACS Flow Cytometer (BD LSRFortessa SORP).

\section{Cell Survival Assay}

Cells were transfected with siRNA for $24 \mathrm{~h}$ then seeded in 6well plates in triplicates at the density of $10^{\wedge 5}$ cells per well in $2 \mathrm{ml}$ medium supplemented with $10 \%$ FBS. Cells were added with $100 \mu \mathrm{M} \mathrm{H}_{2} \mathrm{O}_{2}$ after fixing on the plate, then incubated for $24 \mathrm{~h}$. For trypan blue staining, cells were harvested with trypsin, washed once and resuspended in PBS, then added $10 \mu \mathrm{l}$ trypan blue into cell suspension at volume ratio 1:1. Live cell ratio was determined by counting using a hemocytometer. For CV staining, cells were fixed with $10 \%$ formalin for $5 \mathrm{~min}$ and 
stained with $0.05 \% \mathrm{CV}$ for $30 \mathrm{~min}$. After washed with distilled water, cells were photographed.

\section{Soft Agar and Colony Formation Assay}

U87 cells were transfected with Luciferase siRNA or CREB1 and ATF1 siRNA for $24 \mathrm{~h}$ and then suspended in $1 \mathrm{ml}$ MEM medium supplemented with or without $100 \mu \mathrm{M} \mathrm{H}_{2} \mathrm{O}_{2}$ plus $20 \%$ FBS containing $0.3 \%$ agarose and plated on the firm $0.6 \%$ agarose base in the 12 -well plates (2,000 cells per well). Cells were then cultured in a $37^{\circ} \mathrm{C}$ with $5 \% \mathrm{CO}_{2}$ incubator. After 23 weeks, colonies were stained with $0.05 \%$ crystal violet in PBS for $1 \mathrm{~h}$. Counted under a microscope and photoed after colonies turning into blue.

\section{Statistical Analysis}

All experiments in this work were performed at least three times independently. Multiple groups comparison was analyzed by two-way ANOVA with multiple comparison test by GraphPad Prism 7. For experiments with only two groups, a two-tailed Student's $t$-test was used to calculate the $P$-value. $P<0.05$ was considered significant. ${ }^{*} p<0.05,{ }^{* *} p<0.01,{ }^{* * *} p<0.001$, $* * * * p<0.0001$, NS, not significant.

\section{DATA AVAILABILITY STATEMENT}

The raw data supporting the conclusions of this article will be made available by the authors, without undue reservation.

\section{REFERENCES}

Aggarwal, S., Kim, S. W., Ryu, S. H., Chung, W. C., and Koo, J. S. (2008). Growth suppression of lung cancer cells by targeting cyclic AMP response elementbinding protein. Cancer Res. 68, 981-988. doi: 10.1158/0008-5472.can-060249

Ahmad, I. M., Aykin-Burns, N., Sim, J. E., Walsh, S. A., Higashikubo, R., Buettner, G. R., et al. (2005). Mitochondrial O2* - and $\mathrm{H} 2 \mathrm{O} 2$ mediate glucose deprivationinduced stress in human cancer cells. J. Biol. Chem. 280, 4254-4263. doi: $10.1074 /$ jbc.m411662200

Aykin-Burns, N., Ahmad, I. M., Zhu, Y., Oberley, L. W., and Spitz, D. R. (2009). Increased levels of superoxide and $\mathrm{H} 2 \mathrm{O} 2$ mediate the differential susceptibility of cancer cells versus normal cells to glucose deprivation. Biochem. J. 418, 29-37. doi: $10.1042 /$ bj20081258

Bonni, A., and Brunet, A. (1999). Cell survival promoted by the Ras-MAPK signaling pathway by transcription-dependent and -independent mechanisms. Science 286, 1358-1362. doi: 10.1126/science.286.5443.1358

Chhabra, A., Fernando, H., Watkins, G., Mansel, R. E., and Jiang, W. G. (2007). Expression of transcription factor CREB1 in human breast cancer and its correlation with prognosis. Oncol. Rep. 18, 953-958.

Huang, C. S., Anderson, M. E., and Meister, A. (1993a). Amino acid sequence and function of the light subunit of rat kidney gamma-glutamylcysteine synthetase. J. Biol. Chem. 268, 20578-20583. doi: 10.1016/s0021-9258(20) 80764-9

Huang, C. S., Chang, L. S., Anderson, M. E., and Meister, A. (1993b). Catalytic and regulatory properties of the heavy subunit of rat kidney gammaglutamylcysteine synthetase. J. Biol. Chem. 268, 19675-19680. doi: 10.1016/ s0021-9258(19)36569-x

Jiang, P., Du, W., Wang, X., Mancuso, A., Gao, X., Wu, M., et al. (2011). p53 regulates biosynthesis through direct inactivation of glucose-6-phosphate dehydrogenase. Nat. Cell Biol. 13, 310-316. doi: 10.1038/ncb2172

\section{AUTHOR CONTRIBUTIONS}

LZ, WX, and PJ designed the experiments. LZ and WX performed all the experiments. LZ and PJ wrote the manuscript. All authors reviewed and commented on the manuscript.

\section{FUNDING}

This research was supported by the National Key R\&D Program of China (2019YFA0801701), Tsinghua-Peking Center for Life Sciences, and National Natural Science Foundation of China (81930082) to PJ.

\section{ACKNOWLEDGMENTS}

We thank Xiaohui Liu, Xueying Wang, and Lina Xu for the help with LC-MS analysis. We also thank all of the members of the Jiang laboratory for their technical assistance and/or discussions.

\section{SUPPLEMENTARY MATERIAL}

The Supplementary Material for this article can be found online at: https://www.frontiersin.org/articles/10.3389/fcell.2021. 698264/full\#supplementary-material

Krejsa, C. M., Franklin, C. C., White, C. C., Ledbetter, J. A., Schieven, G. L., and Kavanagh, T. J. (2010). Rapid activation of glutamate cysteine ligase following oxidative stress. J. Biol. Chem. 285, 16116-16124. doi: 10.1074/jbc.m110.1 16210

Li, H., Stokes, W., Chater, E., Roy, R., de Bruin, E., Hu, Y., et al. (2016). Decreased glutathione biosynthesis contributes to EGFR T790M-driven erlotinib resistance in non-small cell lung cancer. Cell Discov. 2:16031.

Lu, S. C. (2009). Regulation of glutathione synthesis. Mol. Aspects Med. 30, 42-59. doi: 10.1016/j.mam.2008.05.005

Mayr, B., and Montminy, M. (2001). Transcriptional regulation by the phosphorylation-dependent factor CREB. Nat. Rev. Mol. Cell Biol. 2, 599-609. doi: $10.1038 / 35085068$

Pigazzi, M., Ricotti, E., Germano, G., Faggian, D., Aricò, M., and Basso, G. (2007). cAMP response element binding protein (CREB) overexpression CREB has been described as critical for leukemia progression. Haematologica 92, 1435-1437. doi: 10.3324/haematol.11122

Quintana-Cabrera, R., Fernandez-Fernandez, S., Bobo-Jimenez, V., Escobar, J., Sastre, J., Almeida, A., et al. (2012). gamma-Glutamylcysteine detoxifies reactive oxygen species by acting as glutathione peroxidase-1 cofactor. Nat. Commun. 3:718.

Rodic, S., and Vincent, M. D. (2018). Reactive oxygen species (ROS) are a key determinant of cancer's metabolic phenotype. Int. J. Cancer 142, 440-448. doi: 10.1002/ijc.31069

Seelig, G. F., Simondsen, R. P., and Meister, A. (1984). Reversible dissociation of gamma-glutamylcysteine synthetase into two subunits. J. Biol. Chem. 259, 9345-9347. doi: 10.1016/s0021-9258(17) 42703-7

Shankar, D. B., Cheng, J. C., Kinjo, K., Federman, N., Moore, T. B., Gill, A., et al. (2005). The role of CREB as a proto-oncogene in hematopoiesis and in acute myeloid leukemia. Cancer Cell 7, 351-362. doi: 10.1016/j.ccr.2005. 02.018 
Shukla, A., Bosenberg, M. W., MacPherson, M. B., Butnor, K. J., Heintz, N. H., Pass, H. I., et al. (2009). Activated cAMP response element binding protein is overexpressed in human mesotheliomas and inhibits apoptosis. Am. J. Pathol. 175, 2197-2206. doi: 10.2353/ajpath.2009.090400

Thway, K., and Fisher, C. (2012). Tumors with EWSR1-CREB1 and EWSR1-ATF1 fusions: the current status. Am. J. Surg. Pathol. 36, e1-e11.

Veeravalli, K., Boyd, D., Iverson, B. L., Beckwith, J., and Georgiou, G. (2011). Laboratory evolution of glutathione biosynthesis reveals natural compensatory pathways. Nat. Chem. Biol. 7, 101-105. doi: 10.1038/nchembi 0.499
Conflict of Interest: The authors declare that the research was conducted in the absence of any commercial or financial relationships that could be construed as a potential conflict of interest.

Copyright (c) 2021 Zhao, Xia and Jiang. This is an open-access article distributed under the terms of the Creative Commons Attribution License (CC BY). The use, distribution or reproduction in other forums is permitted, provided the original author(s) and the copyright owner(s) are credited and that the original publication in this journal is cited, in accordance with accepted academic practice. No use, distribution or reproduction is permitted which does not comply with these terms. 